\title{
Zhilong Huoxue Tongyu Capsule Alleviated the Pyroptosis of Vascular Endothelial Cells Induced by ox-LDL through miR-30b-5p/NLRP3
}

\author{
Mengnan Liu $\mathbb{D}^{\mathbb{D}},{ }^{1,2}$ Gang Luo ${ }^{\mathbb{D}},{ }^{1}$ Tianzhu Liu $\mathbb{D}^{\mathrm{D}},{ }^{3}$ Tingfu Yang ${ }^{\mathbb{D}},{ }^{1}$ Raoqiong Wang $\mathbb{D}^{2,4}$ \\ Wei Ren $\mathbb{D}^{5},{ }^{5}$ Ping Liu $\mathbb{D}^{1},{ }^{1}$ Xiaoling Lai $\mathbb{D},{ }^{1}$ Hua Zhou $\mathbb{D}^{2,6,7}$ and Sijin Yang $\mathbb{D}^{1,2}$ \\ ${ }^{1}$ National Traditional Chinese Medicine Clinical Research Base and Department of Cardiovascular, \\ Hospital (T.C.M) Affiliated to Southwest Medical University, Luzhou, Sichuan, China \\ ${ }^{2}$ Faculty of Chinese Medicine and State Key Laboratory of Quality Research in Chinese Medicine, \\ Macau University of Science and Technology, Taipa, Macao, China \\ ${ }^{3}$ Department of Neurology, Hospital (T.C.M) Affiliated to Southwest Medical University, Luzhou, Sichuan, China \\ ${ }^{4}$ Department of Scientific Research, Hospital (T.C.M) Affiliated to Southwest Medical University, Luzhou, Sichuan, China \\ ${ }^{5}$ Drug Research Center of Integrated Traditional Chinese and Western Medicine, \\ Hospital (T.C.M) Affiliated to Southwest Medical University, Luzhou, Sichuan, China \\ ${ }^{6}$ Zhuhai Hospital of Integrated Traditional Chinese and Western Medicine, Zhuhai, Guangdong, China \\ ${ }^{7}$ Joint Laboratory for Translational Cancer Research of Chinese Medicine of the Ministry of Education of the People's Republic of \\ China, Guangzhou University of Chinese Medicine, Guangzhou, Guangdong, China
}

Correspondence should be addressed to Sijin Yang; ysjimn@sina.com

Received 15 July 2021; Revised 22 November 2021; Accepted 23 November 2021; Published 27 January 2022

Academic Editor: Daniel Dias Rufino Arcanjo

Copyright (C) 2022 Mengnan Liu et al. This is an open access article distributed under the Creative Commons Attribution License, which permits unrestricted use, distribution, and reproduction in any medium, provided the original work is properly cited.

Background. Our previous studies have demonstrated a protective role of Zhilong Huoxue Tongyu capsule in atherosclerosis (AS); however, the molecular mechanisms are unclear. Methods. Human coronary artery endothelial cells (HCAECs) were induced with oxidized low-density lipoprotein (ox-LDL) to obtain cellular AS models. Then, the medicated serum of Zhilong Huoxue Tongyu capsule was obtained and used for treatment with ox-LDL-induced HCAECs. The cell viability was detected by CCK- 8 assay. Besides, the binding between miR-30b-5p and NLRP3 was determined by the dual-luciferase reporter gene system assay. Furthermore, oxLDL-induced HCAECs were transfected with miR-30b-5p mimic or miR-30b-5p inhibitor. The pyroptosis of HCAECs was assessed by flow cytometry, LDH content detection, and qRT-PCR assays. Results. 10\% medicated serum of Zhilong Huoxue Tongyu capsule was the maximum nontoxic concentration and it was used in subsequent assays. The rate of pyroptosis, LDH content, and the mRNA expression level of pyroptosis-related genes including NLRP3, ASC, Caspase 1, IL-1 $\beta$, and IL-18 were prominently enhanced after HCAECs were induced by ox-LDL, which were markedly rescued with medicated serum of Zhilong Huoxue Tongyu capsule. In addition, the medicated serum of Zhilong Huoxue Tongyu capsule significantly enhanced the ox-LDL-induced reduction of miR30b-5p level. NLRP3 could bind to miR-30b-5p and was negatively corrected with miR-30b-5p. Moreover, all the rates of pyroptosis, LDH content, and the mRNA expression levels of pyroptosis-related genes including NLRP3, ASC, Caspase 1, IL- $1 \beta$, and IL-18 were further observably decreased after ox-LDL-induced HCAECs treated with medicated serum were transfected with miR-30b-5p mimic, while these were significantly rescued with transfection of miR-30b-5p inhibitor. Conclusion. Zhilong Huoxue Tongyu capsule alleviated the pyroptosis of vascular endothelial cells induced by ox-LDL through miR-30b-5p/NLRP3. 


\section{Introduction}

Atherosclerosis (AS) is the main cause of a variety of cardiovascular and cerebrovascular diseases, including coronary heart disease and stroke, which has brought about huge medical and economic burdens to society and individuals [1]. The occurrence and development of AS involve multiple factors. Among them, pathological factors such as smoking, hypertension, hyperlipidemia, hyperuric acid, and hyperglycemia causing vascular endothelial damage through inflammatory and oxidative stress responses are the initiating link of AS [2-4]. Thus, AS is a chronic inflammatory progress that is characterized by atherosclerotic plaques, vascular stenosis, inflammation, and lipid metabolism $[5,6]$. Oxidative low-density lipoprotein (ox-LDL) plays a key role in atherosclerotic plaque formation and endothelial cell damage. After endothelial damage caused by ox-LDL, it can promote a variety of inflammatory cells to infiltrate the damaged vascular endothelium. Also, foam cells are formed and gradually accumulate to form atherosclerotic plaques after inflammatory cells engulf ox-LDL [7, 8]. Cell death caused by inflammation is closely related to cell pyroptosis [9]. Therefore, protecting endothelial cell pyrolysis caused by ox-LDL is considered to be a key target for treating AS and reducing the incidence and mortality of cardiovascular and cerebrovascular diseases.

Pyroptosis is a highly proinflammatory programmed cell death. Activated caspases cleave the pore-forming protein Gasdermin D (GSDMD) to rupture the plasma membrane, leading to the release of proinflammatory factors interleukin- $1 \beta$ (IL-1 $\beta$ ) and IL-18 [10]. The assembly of the inflammasome is the first step in the beginning of cell pyrolysis, of which the inflammasome, such as NLRP3 inflammasome, binds to pattern recognition receptors to complete assembly [11]. Moreover, NLRP3 inflammasomes have been demonstrated to play an important role in the occurrence and development of AS. Duewell et al. reported that cholesterol crystals activate NLRP3 inflammasomes to promote pyroptosis of vascular endothelial cells, which leads to endothelial dysfunction to induce the occurrence of AS [12]. Furthermore, microRNAs (miRNAs), a category of endogenous, evolutionarily conserved, and small noncoding RNA sequences $[13,14]$, have been found to regulate the progress of pyroptosis via targeting miRNAs $[15,16]$. Thus, the pyroptosis of vascular endothelial cells may be modulated via the miRNAs/NLRP3 signaling axis, which exerts a decisive and important role in the occurrence and development of AS.

Zhilong Huoxue Tongyu capsule is a pure Chinese medicine in-hospital preparation developed by Professor Yang Sijin of our hospital which is mainly developed from traditional Chinese medicines including Astragalus, Pheretima, Caulis Sargentodoxae, Cassia Twig, and Hirudo. Our group has demonstrated the outstanding effectiveness of Zhilong Huoxue Tongyu capsule on the ischemic stroke and related diseases, and its mechanism of action may be related to lowering blood lipids and inhibiting inflammation [17-19]. In addition, we also report that Zhilong Huoxue Tongyu capsule can improve the metabolism of serum lipids and reduce the formation of carotid atherosclerotic plaques in hyperlipidemia and carotid atherosclerosis rabbits, which may be associated with the inhibition of NLRP3 inflammasome activation and downstream inflammatory factors release by suppressing the phosphorylation of NF- $\kappa \mathrm{B}$. Thus, we concluded that Zhilong Huoxue Tongyu capsule exhibited the antiatherosclerosis effect through NLRP3 inflammasome signaling pathway [20]. More importantly, recent study has shown that miR-30b-5p is negatively correlated with NLRP3 in mouse and human liver, and treatment of miR-30b-5p agomir can target NLRP3 and relieve liver inflammation in the injured liver [21]. Taken together, we speculated that the medicated serum of Zhilong Huoxue Tongyu capsule alleviated the pyroptosis of vascular endothelial cells induced by ox-LDL through miR-30b-5p/ NLRP3.

The present study reports observable effectiveness of Zhilong Huoxue Tongyu capsule on the pyroptosis of vascular endothelial cells induced by ox-LDL in vitro. Mechanistically, Zhilong Huoxue Tongyu capsule alleviated the pyroptosis of vascular endothelial cells induced by ox-LDL through miR-30b-5p/NLRP3. The results of this study will provide new insights and methods for the therapy of AS and even other inflammation-related cardiovascular and cerebrovascular diseases.

\section{Materials and Methods}

2.1. Animal. Adult Sprague Dawley rats (age: 7-8 weeks, weight: $200-220 \mathrm{~g}$ ) were purchased and acclimated to standard laboratory conditions for 7 days before experiments. Rats were provided with a 12 hour $/ 12 \mathrm{~h}$ light-dark cycle and fed with standard diet and water ad libitum at $(25 \pm 2)^{\circ} \mathrm{C}$ and $40 \%-60 \%$ the relative humidity. All the procedures were carried out strictly based on the National Institute of Health Guide for the Care and Use of Laboratory Animals. Also, the study was ratified by the Board and Ethics Committee of Hospital (T.C.M) Affiliated to Southwest Medical University.

2.2. Cell Culture. Human coronary artery endothelial cells (HCAECs) were purchased from Procell (CP-H087, Wuhan, China) and cultured in complete medium for human umbilical vein endothelial cells (CM-H087, Wuhan, China) at $37^{\circ} \mathrm{C}$ with $5 \%$ carbon dioxide $\left(\mathrm{CO}_{2}\right)$.

2.3. Cell Transfection. miR-30b-5p mimics, miR-30b-5p inhibitor, and the corresponding negative controls (NC) were designed and synthesized by Ribobio (Guangzhou, China). It was performed with RiboFect ${ }^{\mathrm{TM}} \mathrm{CP}$ Transfection Kit (C10511-05, Ribobio) based on the experimental procedures for the following assays.

2.4. Preparation of Medicated Serum. For in vitro experiments, medicated serum was first prepared with the following description. 20 rats were randomly divided into two groups $(n=5)$ : control and Zhilong Huoxue Tongyu capsule 
group. Rats in Zhilong Huoxue Tongyu capsule group were intragastrically administered with $2.52 \mathrm{~g} /(\mathrm{kg} . \mathrm{d})$ Zhilong Huoxue Tongyu capsule, while rats in control group were intragastrically administered with $1 \mathrm{ml} / 100 \mathrm{~g}$ saline a day for 5 consecutive days. Then, blood was taken from the abdominal aorta after the rats were intraperitoneally anesthetized with sodium pentobarbital $(40 \mathrm{mg} / \mathrm{kg})$. Serum was isolated and the complement was inactivated for in vitro experiments.

2.5. Experimental Groups and Drug Administration. HCAECs were inoculated into six-well plates and divided into six groups: control, ox-LDL, ox-LDL + blank serum, ox-LDL + medicated serum (MS), ox-LDL + MS + miR30b-5p-mimic, and ox-LDL + MS + miR-30b-5p-inhibitor. HCAECs in control group were cultured in complete medium for human umbilical vein endothelial cells, while cells in the other five groups were cultured in complete medium for human umbilical vein endothelial cells including $50 \mathrm{nM}$ ox-LDL for $24 \mathrm{~h}$ [22]. Subsequently, cells in ox-LDL + blank serum group were cultured in complete medium for human umbilical vein endothelial cells including $200 \mu \mathrm{L}$ blank serum obtained from control rats, while cells in ox-LDL + medicated serum (MS), ox$\mathrm{LDL}+\mathrm{MS}+\mathrm{miR}-30 \mathrm{~b}-5 \mathrm{p}-\mathrm{mimic}$, and ox-LDL + MS + miR30b-5p-inhibitor were cultured in complete medium for human umbilical vein endothelial cells including $200 \mu \mathrm{L}$ medicated serum obtained from rats in Zhilong Huoxue Tongyu capsule group described in Section 2.3 above. Cells in control group and ox-LDL group were cultured in complete medium for human umbilical vein endothelial cells supplied with $200 \mu \mathrm{L}$ phosphate buffer saline (PBS, 201201A17, Yuanmu Biological Technology, Shanghai, China). Next, cells in ox-LDL + MS + miR-30b-5p-mimic and ox-LDL + MS + miR-30b-5p-inhibitor groups were transfected $5 \mathrm{nM}$ miR-30b-5p-mimic and miR-30b-5p-inhibitor, respectively. Cells were maintained at $37^{\circ} \mathrm{C}$ with $5 \%$ $\mathrm{CO}_{2}$ for further $24 \mathrm{~h}$.

2.6. Cell Counting Kit-8 Assay. HCAECs were inoculated in 96-well plates with a density of $1 \times 10^{5} /$ well and cultured for $24 \mathrm{~h}$ at $37^{\circ} \mathrm{C}$ in $5 \% \mathrm{CO}_{2}$. Subsequently, the Cell Count Kit-8 (Dojindo Laboratories, Kumamoto, Japan) was used to detect the proliferation of cells according to the operating manual. The absorbance was recorded at $450 \mathrm{~nm}$ by a microplate reader (Thermo Fisher Scientific, Waltham, MA, USA).

2.7. Flow Cytometry. The pyroptosis of HCAECs was determined using pyroptosis/Caspase 1 cell pyroptosis detection kit (9145, ImmunoChemistry Technologies, USA). Cells were collected and resuspended with $500 \mu$ l Binding Buffer and then stained with a mixture of $5 \mu$ FAM-YVADFMK-FLICA and $5 \mu \mathrm{l}$ propidium iodide (PI) (SigmaAldrich) for $15 \mathrm{~min}$ at room temperature in the dark. The rate of pyroptosis was examined by flow cytometry (BD FACSVerse, USA).
2.8. Quantitative Reverse Transcriptase-Polymerase Chain Reaction ( $q R T-P C R)$. Total RNA was extracted from cell samples using TRIzol reagent (TaKaRa Biotechnology Co., Ltd., Dalian, China) according to the manufacturer's specifications. cDNA was synthesized with a PrimeScript RT Reagent Kit (Takara, RR047A) in line with the manufacturer's instruction. qRT-PCR was carried out by the Bio-Rad Scrip $^{\mathrm{TM}}$ cDNA Synthesis Kit (Bio-Rad Laboratories, Inc., Hercules, CA, USA). The primer sequences were designed and synthesized. The qRT-PCR amplification conditions were as follows: $95^{\circ} \mathrm{C}$ for $5 \mathrm{~min}, 95^{\circ} \mathrm{C}$ for $15 \mathrm{~s}$, and $60^{\circ} \mathrm{C}$ for $30 \mathrm{~s}$ of 40 cycles. $\beta$-actin served as the internal control. For the quantification of $\mathrm{miR}-30 \mathrm{~b}-5 \mathrm{p}$ expression, the RT reaction was performed using Bulge-Loop ${ }^{\text {TM }}$ miRNA RT-qPCR Primer (RiboBio Co., Ltd., Guangzhou, China). The RT reaction was processed at $42^{\circ} \mathrm{C}$ for $60 \mathrm{~min}$ and at $70^{\circ} \mathrm{C}$ for $10 \mathrm{~min}$. The $\mathrm{miR}-30 \mathrm{~b}-5 \mathrm{p}$ expression level was quantified at $95^{\circ} \mathrm{C}$ for $10 \mathrm{~min}$, followed by 40 cycles at $95^{\circ} \mathrm{C}$ for $2 \mathrm{sec}, 60^{\circ} \mathrm{C}$ for $20 \mathrm{sec}$, and $70^{\circ} \mathrm{C}$ for $10 \mathrm{sec}$. U6 served as the internal control. The level of genes was analyzed by the comparative threshold cycle method $\left(2^{-\Delta \Delta C T}\right.$ method), where $\Delta \Delta \mathrm{CT}=\Delta \mathrm{CT}_{\text {treatment }}-\Delta \mathrm{CT}_{\text {control }}$ and $\Delta \mathrm{CT}=C t_{\text {target }}-$ $C t_{\text {reference }}$. The primer sequences used in the present study are listed as follows: NLRP3: forward primer: $5^{\prime}$ TCTCAGCACCAACCAGAGCCTCAC- $3^{\prime}$, reverse primer: 5'-CCACGCACAGCAGTCTGACTCCAA- ${ }^{\prime}$; ASC: forward primer: 5'-TGGACCAACACAGGCAAGCACTCA$3^{\prime}$, reverse primer: 5'-AGGTCAGGTTCCAGGCTGGAGCAA-3'; Caspase 1: forward primer: $5^{\prime}$-CCTGGCAGGAATTCTGGAGCTTCAATC- $3^{\prime}$, reverse primer: $5^{\prime}$ GGCAAGACGTGTACGAGTGGTTGTATT-3'; IL-1 $\beta$ : forward primer: $5^{\prime}$-ATCCTCTCCAGTCAGGCTTCCTT GTG - $3^{\prime}$, reverse primer: $5^{\prime}$-AGCTCTTGTCGAGATGCTGCTGTGA-3'; IL-18: forward primer: $5^{\prime}$ TGCCTGATATCGACCGAACAGCCAAC - $3^{\prime}$, reverse primer: $\quad 5^{\prime}$-ACAGATAGGGTCACAGCCAGTCCTCT-3'; $\beta$-actin: forward primer: $5^{\prime}$-CTGGCACCACACCTTCTA CA-3', reverse primer: $5^{\prime}$-GGGTCATCTTCTCACGGTTG3'; miR-30b-5p: forward primer: $5^{\prime}$-ACGGGCAAAAATACTCCAGCTCTCAAT- $3^{\prime}$, reverse primer: $5^{\prime}$ CTCTGGAAAACTGGTGTCGACTGGTGTC-3'; and U6: forward primer: 5'-GCTTCGGCAGCACATATACTAAA AT $-3^{\prime}$, reverse primer: 5'-CGCTTCACGAATTTGC GTGTCAT $-3^{\prime}$.

2.9. Detection of $\mathrm{LDH}$. The content of $\mathrm{LDH}$ was detected using LDH assay kit (A020-1, NJJCBIO, Nanjing, China) according to the operating manual. The absorbance was recorded at $440 \mathrm{~nm}$ by a microplate reader (Thermo Fisher Scientific).

2.10. Luciferase Assay. Wild-type and mutant NLRP3 were constructed into pGL3-Basic luciferase vector (Promega, Madison, WI, USA) to produce luciferase reporter plasmids. HCAECs were transfected with the plasmids and then were cotransfected with miR-NC or miR-30b-5p mimic with Lipofectamine 2000 (Life Technologies, New York, USA). The luciferase activity was determined by 
Promega Kit (Promega) after cells were transfected for $48 \mathrm{~h}$.

2.11. Statistical Analysis. Data were shown as the means \pm SE. Shapiro-Wilk test was employed to test continuous quantitative data distribution normality. Student's $t$-test was employed to analyze the data with only two groups, whereas the one-way analysis of variance was used to analyze the differences among multiple groups by the SPSS 22.0 statistical software (IBM, Armonk, New York, USA) followed by post hoc Bonferroni test. The differences were regarded as statistically significant when $p<0.05$.

\section{Results}

3.1. The Effect of Medicated Serum of Zhilong Huoxue Tongyu Capsule on HCAECs Viability. HCAECs were hatched with different concentrations of medicated serum of Zhilong Huoxue Tongyu capsule for $24 \mathrm{~h}(0,5,10,15,20$, and $25 \%)$. The cell viability of HCAECs was dose-dependently reduced with medicated serum of Zhilong Huoxue Tongyu capsule. The maximum nontoxic concentration of medicated serum of Zhilong Huoxue Tongyu capsule was 10\% (Figure 1). Thus, $10 \%$ medicated serum of Zhilong Huoxue Tongyu capsule was used in subsequent assays.

Then, in order to evaluate the effect of medicated serum of Zhilong Huoxue Tongyu capsule on the pyroptosis, AS cell models were induced by ox-LDL. Flow cytometry assays exhibited that the rate of pyroptosis was prominently enhanced after HCAECs were induced by ox-LDL, which was markedly rescued with medicated serum of Zhilong Huoxue Tongyu capsule, not blank serum (Figures 2(a) and 2(b)). Similarly, medicated serum of Zhilong Huoxue Tongyu capsule, not blank serum, notably reduced the ox-LDL-induced elevation of LDH content (Figure 2(c)). Besides, qRTPCR results exhibited that all the mRNA expression levels of pyroptosis-related genes including NLRP3, ASC, Caspase 1, IL- $1 \beta$, and IL-18 were significantly increased after HCAECs were induced by ox-LDL, which was markedly reversed with medicated serum of Zhilong Huoxue Tongyu capsule, not blank serum (Figure 2(d)). Therefore, these results indicated that the medicated serum of Zhilong Huoxue Tongyu capsule relieved the pyroptosis of HCAECs induced by oxLDL in vitro.

3.2. NLRP3 Binds to $m i R-30 b-5 p$. In addition, the level of miR-30b-5p was significantly reduced after HCAECs were induced by ox-LDL, which was notably antagonized with medicated serum of Zhilong Huoxue Tongyu capsule, not blank serum (Figure 3(a)). To further explore the underlying molecular mechanism of miR-30b-5p, an NLRP3 wild-type plasmid (NLRP3-WT) or an NLRP3 mutant plasmid (NLRP3-MUT) was constructed for the luciferase reporter assay (Figure 3(b)). The results showed that the luciferase activity of NLRP3-WT reporter plasmids was dramatically inhibited compared to that in the negative control (NC) in HCAECs. However, no statistical difference was observed in the luciferase activity of NLRP3 compared to that in the NC

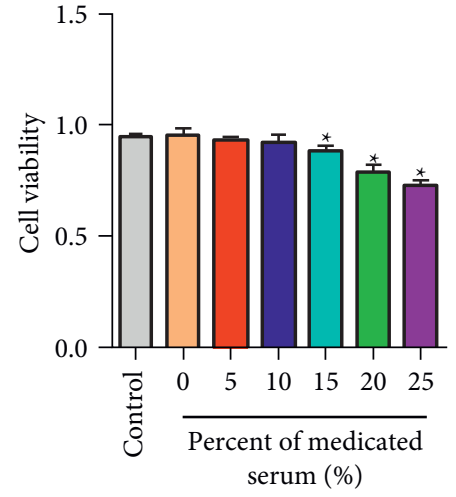

Figure 1: The effect of medicated serum of Zhilong Huoxue Tongyu capsule on HCAECs viability. HCAECs were hatched with different concentrations of medicated serum of Zhilong Huoxue Tongyu capsule for $24 \mathrm{~h}(0,5,10,15,20$, and $25 \%)$. The cell viability was detected by CCK- 8 Kit. Data of four independent samples were shown as mean \pm SE. ${ }^{*} P<0.05$. The medicated serum of Zhilong Huoxue Tongyu capsule relieves the pyroptosis of HCAECs induced by ox-LDL in vitro.

in HCAECs (Figure 3(c)). Moreover, miR-30b-5p mimic prominently inhibited the level of miR-30b-5p, while miR$30 b-5 p$ inhibitor notably elevated the level of miR-30b-5p (Figure 3(d)). Based on these results, we concluded that NLRP3 could bind to miR-30b-5p.

3.3. The Medicated Serum of Zhilong Huoxue Tongyu Capsule Alleviates the Pyroptosis of HCAECs Induced by ox-LDL through $m i R-30 b-5 p / N L R P 3$. The pyroptosis of HCAECs was assessed after ox-LDL-induced HCAECs were transfected with miR-30b-5p mimic or miR-30b-5p inhibitor. The results revealed that all the rates of pyroptosis (Figures 4(a) and 4(b)), LDH content (Figure 4(c)), and the mRNA expression levels of pyroptosis-related genes including NLRP3, ASC, Caspase 1, IL-1 $\beta$, and IL-18 (Figure 4(d)) were further observably decreased after ox-LDL-induced HCAECs treated with medicated serum were transfected with miR30b-5p mimic, while these were significantly rescued with transfection of miR-30b-5p inhibitor. Taken together, these results indicated that the medicated serum of Zhilong Huoxue Tongyu capsule alleviates the pyroptosis of HCAECs induced by ox-LDL through miR-30b-5p/NLRP3.

\section{Discussion}

ox-LDL plays a key role in the pathological process of AS which can act on the vascular endothelium and recruit inflammatory cells, thereby promoting the production of foam cells and the formation of atherosclerotic plaques, leading to the occurrence of endothelial cell pyroptosis $[23,24]$. Current lipid-lowering drugs have achieved the effect of effectively reducing LDL, but how to remove oxLDL is still a difficult problem. Under normal conditions, ox-LDL produced in the body can be quickly cleared by Kupffer cells in the liver [25]. However, continuous lipid metabolism disorder and inflammation in AS lead to 


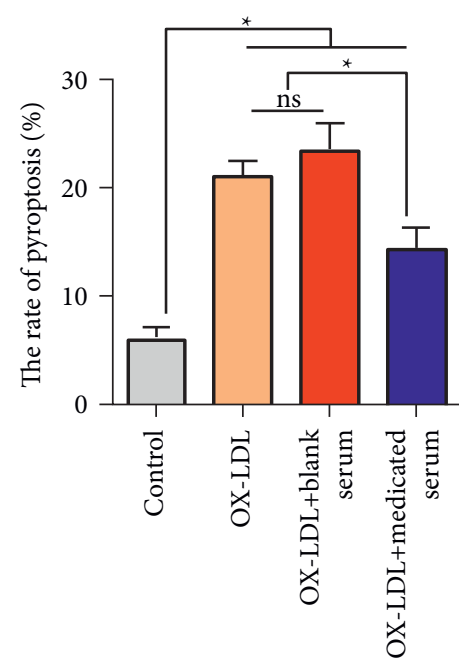

(a)
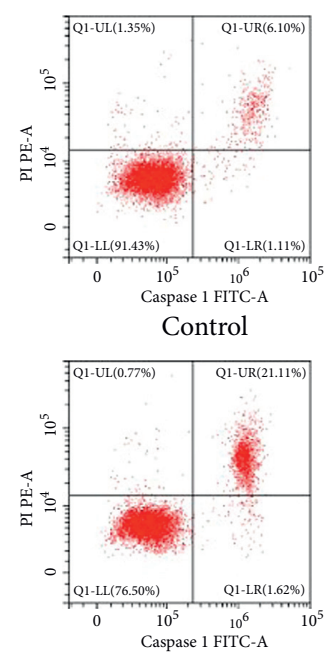

OX-LDL+blank serum
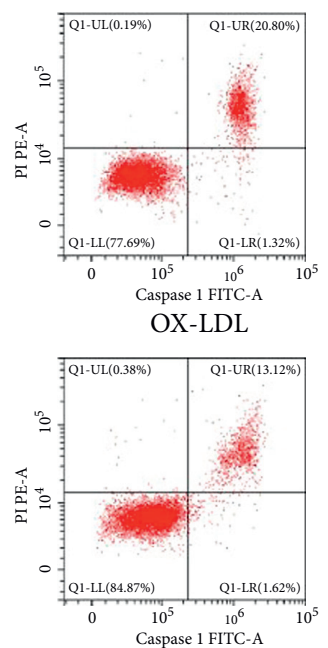

OX-LDL+medicated serum

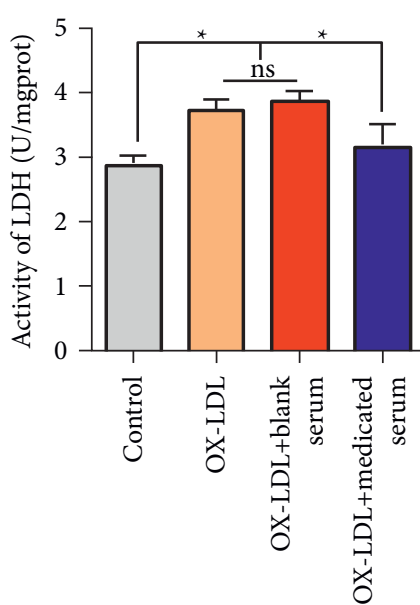

(c)

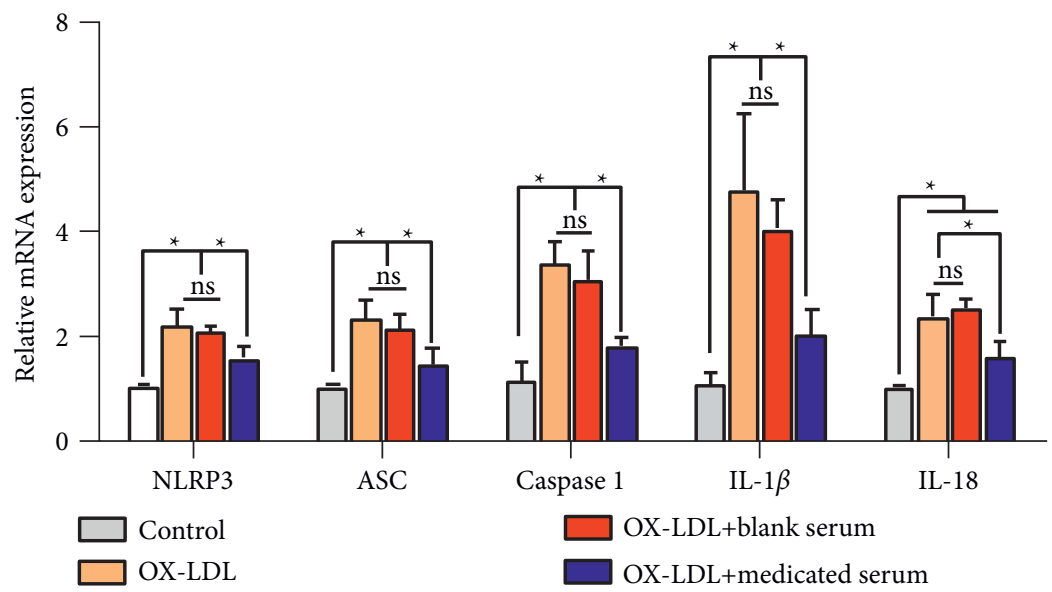

(d)

Figure 2: The medicated serum of Zhilong Huoxue Tongyu capsule relieves the pyroptosis of HCAECs induced by ox-LDL in vitro. HCAECs were treated with $50 \mathrm{nM}$ ox-LDL for $24 \mathrm{~h}$ to induce atherosclerosis cell models and then incubated with $200 \mu \mathrm{L}$ Zhilong Huoxue Tongyu capsule medicated serum. (a, b) The rate of pyroptosis was determined by flow cytometry assays. (c) The LDH content was detected using commercial kit. (d) The relative mRNA levels of NLRP3, ASC, Caspase 1, IL-1 $\beta$, and IL-18 were detected by qRT-PCR assay. The data were expressed after being normalized to $\beta$-actin. Data of three independent samples were shown as mean \pm SE. ${ }^{*} P<0.05$.

increased ox-LDL in the circulation [25]. Therefore, this study mainly explores new therapeutic directions besides lipid lowering and focuses on Zhilong Huoxue Tongyu capsule effectively removing cell pyrosis induced by ox-LDL. Our previous studies have demonstrated a protective role of Zhilong Huoxue Tongyu capsule in the ischemic stroke and related diseases, as well as AS [26, 27].

Modern pharmacology confirmed that the main pharmacological components of Astragalus in Zhilong Huoxue Tongyu capsule are Astragalus total flavonoids, Astragalus polysaccharides, and Astragalus saponins. Astragalus total flavonoids can scavenge oxygen free radicals in vivo. Astragalus polysaccharides (APS) may play a direct antiinflammatory role, inhibit nuclear factor $\kappa \mathrm{B}$ expression, and scavenge free radicals, thereby reducing nerve cell apoptosis and accelerating the recovery of nerve function defects. Hirudin, the main component of leech, is the strongest thrombin inhibitor discovered so far. It combines with the active center of thrombin to form a stable noncovalent bond compound, thus inhibiting the hydrolysis of thrombin to fibrinogen and blocking the coagulation cascade reaction. At the same time, the combination of thrombin and Hirudin could not lyse the extracellular N-terminal of the receptor activated by protease, so the thrombin receptor could not be activated, thus inhibiting the receptor-mediated neuronal apoptosis and stimulating the activation, proliferation, and secretion of cytokines of microglia [28]. In addition, the collateral circulation can be opened by activating blood circulation and removing blood stasis, so that the hematoma can be in contact with the surrounding blood vessels to promote its absorption and improve the surrounding hypoperfusion injury [29]. In the present study, we established the role of Zhilong Huoxue Tongyu capsule in the pyroptosis. The results revealed that Zhilong Huoxue 


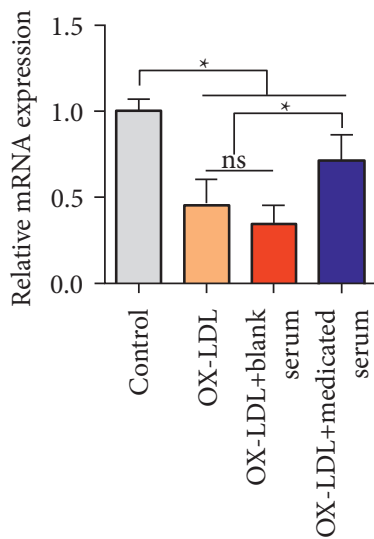

(a)

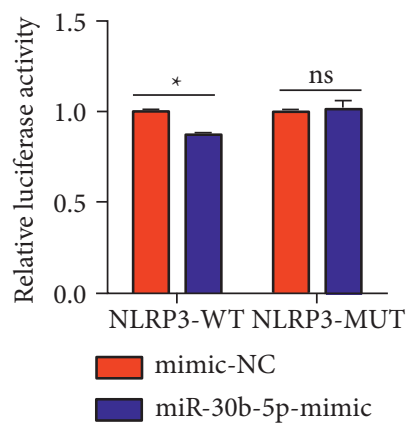

(c)
Position 363-369 of NLRP3 3' UTR 5' ...UUUUCUUCUUUGUUC--UGUUUACU...

hsa-miR-30b-5p

3'

UCGACUCACAUCCUACAAAUGU (b)

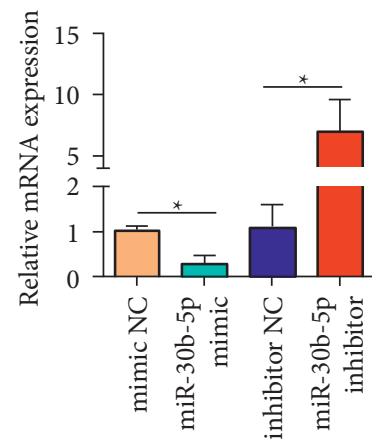

(d)

Figure 3: NLRP3 binds to miR-30b-5p. (a) HCAECs were treated with $50 \mathrm{nM}$ ox-LDL for $24 \mathrm{~h}$ to induce atherosclerosis cell models and then incubated with $200 \mu \mathrm{L}$ Zhilong Huoxue Tongyu capsule medicated serum. The level of miR-30b-5p was detected by qRT-PCR assay. The data were expressed after being normalized to U6. (b) The binding sites of miR-30b-5p and NLRP3 were forecasted via the bioinformatics website. NLRP3 wild-type plasmids and mutant plasmids were constructed. (c) NLRP3 wild-type and mutant plasmids were constructed into pGL3-Basic luciferase vector to produce luciferase reporter plasmids. HCAECs were transfected with the plasmids and then were cotransfected with miR-NC or miR-30b-5p mimic with Lipofectamine 2000; after cells were transfected for $48 \mathrm{~h}$, the direct interaction of NLRP3 and miR-30b-5p was verified by luciferase reporter experiment. (d) The relative mRNA level of NLRP3 was detected by qRT-PCR assay after HCAECs were transfected with miR-30b-5p mimic or miR-30b-5p inhibitor. The data were expressed after being normalized to $\beta$-actin. Data of three independent samples were shown as mean \pm SE. ${ }^{*} P<0.05$.

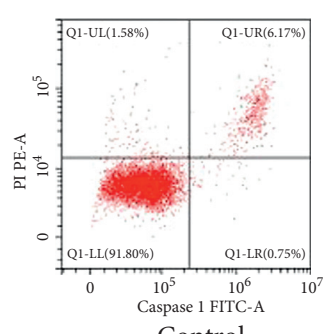

Control

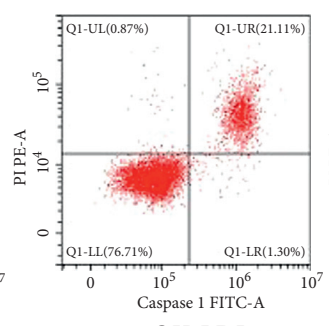

OX-LDL

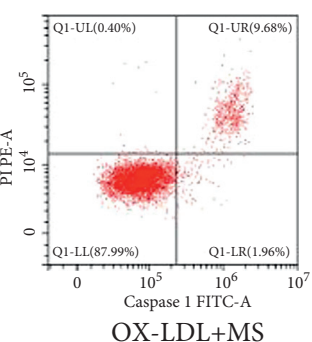

(a)
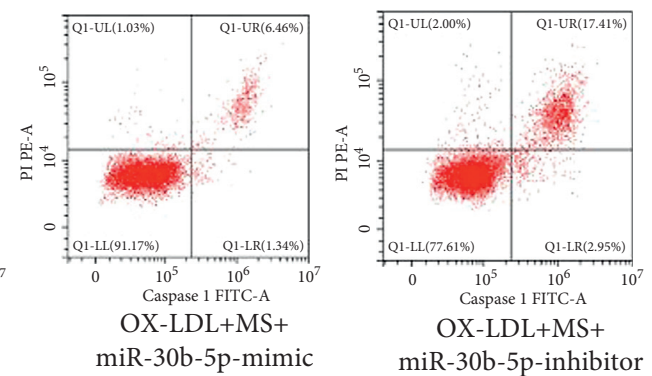

OX-LDL+MS+ miR-30b-5p-inhibitor

FIgUre 4: Continued. 


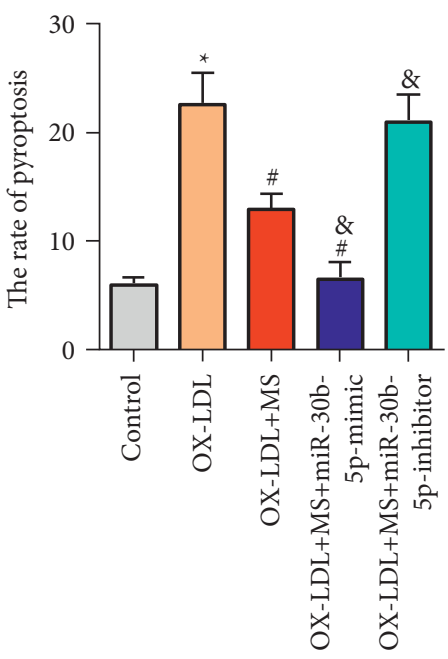

(b)

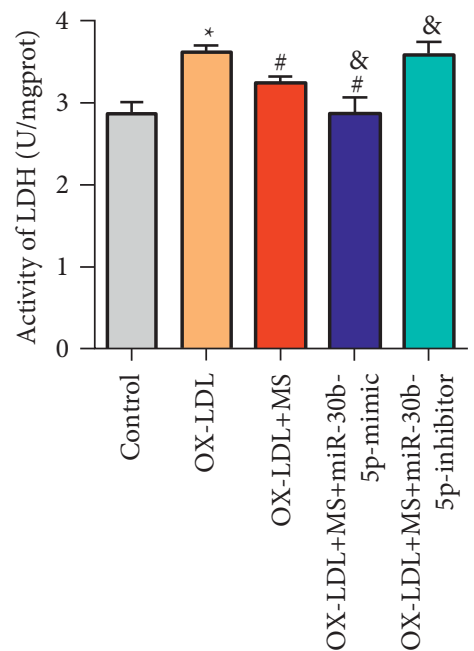

(c)

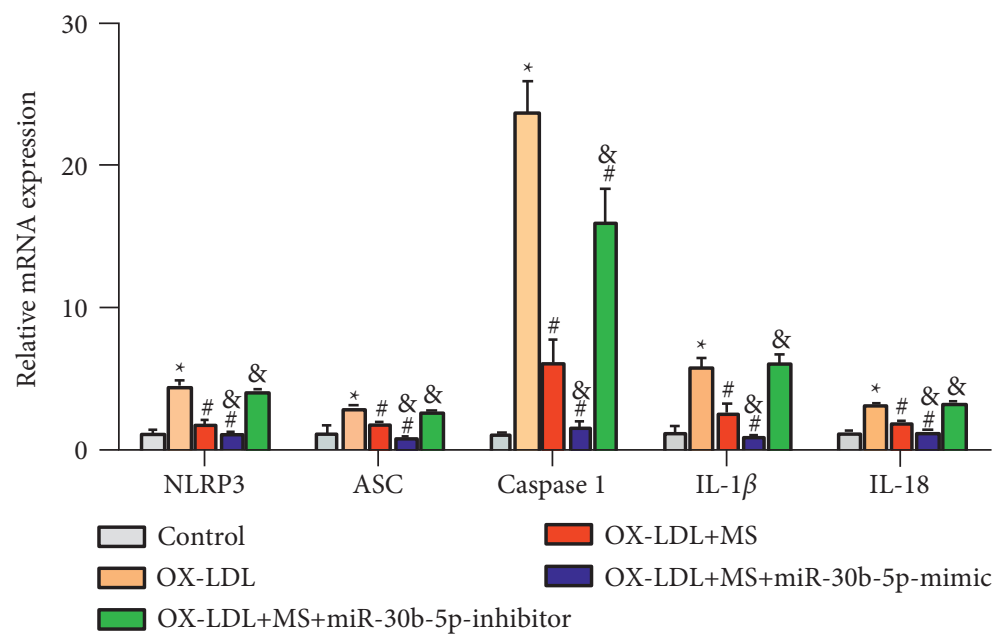

(d)

Figure 4: The medicated serum of Zhilong Huoxue Tongyu capsule alleviates the pyroptosis of HCAECs induced by ox-LDL through miR$30 \mathrm{~b}-5 \mathrm{p} / \mathrm{NLRP} 3$. HCAECs were treated with $50 \mathrm{nM}$ ox-LDL for $24 \mathrm{~h}$ to induce atherosclerosis cell models and then incubated with $200 \mu \mathrm{L}$ Zhilong Huoxue Tongyu capsule medicated serum. Next, cells were transfected with $5 \mathrm{nM}$ miR-30b-5p-mimic and miR-30b-5p-inhibitor, respectively. (a, b) The rate of pyroptosis was determined by flow cytometry assays. (c) The LDH content was detected using commercial kit. (d) The relative mRNA levels of NLRP3, ASC, Caspase 1, IL-1 $\beta$, and IL-18 were detected by qRT-PCR assay. The data were expressed after being normalized to $\beta$-actin. Data of three independent samples were shown as mean \pm SE. ${ }^{*} P<0.05$ compared to control group. ${ }^{\#} P<0.05$ compared to ox-LDL group. ${ }^{\&} P P<0.05$ compared to ox-LDL + MS. MS: medicated serum. 
Tongyu capsule alleviated the pyroptosis of vascular endothelial cells induced by ox-LDL through miR-30b-5p/ NLRP3.

AS is a complicated inflammatory disease, in which endothelial cells, macrophages, and vascular smooth muscle cells (VSMCs) are mainly three types of cells involved in its progression [30-32]. Among them, endothelial cells act on a core role in maintaining the integrity of vessel wall, and the injury of endothelial cell is generally regarded as the initiation of AS [33]. Thus, HCAECs were used in the present study. Inflammation and cell death are basic features in the start and development of AS, and pyroptosis has been demonstrated to be a type of inflammatory cell death and involved in a variety of cardiovascular diseases [34, 35]. Moreover, it has been reported that ox-LDL can induce cell pyroptosis in HCAECs [36], macrophage [37], and VSMCs [38]. Consistently, our results showed that ox-LDL induced the pyroptosis of HCAECs, as evidenced by the elevation of pyroptosis rate, $\mathrm{LDH}$ content, and the mRNA expressions of NLRP3, ASC, Caspase 1, IL-1 $\beta$, and IL-18. Pyroptosis is a form of Caspase 1-dependent cell death, and the Caspase 1 activation is essential [39]. Also, the activation of Caspase 1 needs the participation of inflammasome including NLRP3, in which NLRP3 recruits ASC and accelerates the activation of Caspase 1 [40]. Upon Caspase 1 activation, it carries out its function to process the precursor of the inflammatory cytokines IL- $1 \beta$ and IL- 18 to generate their mature forms IL$1 \beta$ and IL-18 [41]. However, the medicated serum of Zhilong Huoxue Tongyu capsule reduced the ox-LDL-induced enhancement of pyroptosis rate, $\mathrm{LDH}$ content, and the mRNA expressions of NLRP3, ASC, Caspase 1, IL-1 $\beta$, and IL-18. Therefore, these results indicated that Zhilong Huoxue Tongyu capsule alleviates the pyroptosis of HCAECs induced by ox-LDL.

miRNAs are tightly involved in the progression of oxLDL-induced AS [42, 43]. Among them, miR-30b-5p is identified to be a possible diagnostic and therapeutic biomarker for AS due to its dysregulation both in vivo [44] and in vitro [45]. We discovered that the level of miR-30b-5p was notably downregulated after HCAECs were treated with oxLDL, which was prominently rescued by medicated serum of Zhilong Huoxue Tongyu capsule treatment. Thus, the results suggested that Zhilong Huoxue Tongyu capsule alleviates the pyroptosis of HCAECs induced by ox-LDL through upregulating miR-30b-5p expression. The activation of inflammatory response is an important process in the apoptosis and injury of endothelial cells caused by ox-LDL, and oxLDL-induced activation of inflammatory response can directly cause endothelial sclerosis and thus participate in the formation of atherosclerotic plaques [46]. Meanwhile miR$30 \mathrm{~b}-5 \mathrm{p}$ has been implicated in activation of NLRP3 inflammasome in other studies [47]. Importantly, many research studies suggest that NLRP3 is involved in pyrophosis of AS $[12,48]$. Besides, ox-LDL induces the activation of NLRP3 inflammasome in the aorta [49], which is important for the formation of unstable plaques in AS by promoting Caspase 1 activation of cell pyroptosis involved in ox-LDL-induced apoptosis [48]. Furthermore, miR-30b-5p is negatively correlated with NLRP3 [21], which was confirmed by our results including the dual-luciferase reporter gene system results and qRT-PCR results. In order to further explore the detailed mechanisms, UVECs were transfected with miR-30b-5p mimic or miR-30b-5p inhibitor. The results revealed that the rate of pyroptosis, LDH content, and the mRNA expression levels of pyroptosisrelated genes including NLRP3, ASC, Caspase $1, \mathrm{IL}-1 \beta$, and IL-18 were further observably decreased after ox-LDL-induced HCAECs treated with medicated serum were transfected with miR-30b-5p mimic, while these were significantly rescued with transfection of miR-30b-5p inhibitor. Therefore, these results indicated that Zhilong Huoxue Tongyu capsule alleviates the pyroptosis of HCAECs induced by ox-LDL through miR-30b-5p/NLRP3 and also suggested that the increased expression of miR-30b$5 p$ and the inhibition of NLRP3 inflammasome activation may be the molecular mechanism of Zhilong Huoxue Tongyu capsule to reduce the vascular endothelial cells induced by ox-LDL.

However, combined with the findings of various studies on Zhilong Huoxue Tongyu capsule, there are still some problems that need further discussion. For example, in terms of drug metabolism and pharmacokinetics, there is still a lack of studies on Zhilong Huoxue Tongyu capsule in vivo, which makes it difficult to find its active substance basis for the treatment of cardiovascular diseases. In addition, the basic research of Zhilong Huoxue Tongyu capsule was indepth research on the mechanism of AS, lacking the understanding of the overall mechanism of action. Therefore, molecular biological techniques such as genomics, proteomics, and metabolomics, combined with the analysis of active ingredients, can be used to further study the mechanism of its action in preventing and treating common cardiovascular diseases, providing a solid basis for its clinical application.

In conclusion, this present in vitro study reports observable effectiveness of Zhilong Huoxue Tongyu capsule on the pyroptosis of vascular endothelial cells induced by oxLDL. Mechanistically, Zhilong Huoxue Tongyu capsule alleviated the pyroptosis of vascular endothelial cells induced by ox-LDL through miR-30b-5p/NLRP3. Zhilong Huoxue Tongyu capsule can promote the expression of miR-30b-5p and inhibit the activation of NLRP3 inflammasome in vascular endothelial cells induced by ox-LDL. Moreover, miR-30b-5p can directly target NLRP3 in vascular endothelial cells induced by ox-LDL and reduce the activity of NLRP3 inflammasome. The results of this study will provide new insights and methods for the therapy of AS and even other inflammation-related cardiovascular and cerebrovascular diseases.

\section{Data Availability}

The data used or analyzed during the current study are available from the corresponding author.

\section{Conflicts of Interest}

The authors declare that they have no conflicts of interest. 


\section{Authors' Contributions}

Mengnan Liu and Gang Luo contributed equally to this work.

\section{Acknowledgments}

The present study was supported by the National Natural Science Foundation of China (Grant no. 82074378) and the Project of Sichuan Administration of Traditional Chinese Medicine (Grants nos. 2021MS097 and 2020ZD002).

\section{References}

[1] C. P. Nelson, S. E. Hamby, D. Saleheen et al., "Genetically determined height and coronary artery disease," New England Journal of Medicine, vol. 372, no. 17, pp. 1608-1618, 2015.

[2] G. Gallo, G. Pierelli, M. Forte, R. Coluccia, M. Volpe, and S. Rubattu, "Role of oxidative stress in the process of vascular remodeling following coronary revascularization," International Journal of Cardiology, vol. 268, pp. 27-33, 2018.

[3] M. Y. Sun, M. Zhang, S. L. Chen et al., "The influence of hyperlipidemia on endothelial function of FPN1 tek-cre mice and the intervention effect of tetramethylpyrazine," Cellular Physiology and Biochemistry, vol. 47, no. 1, pp. 119-128, 2018.

[4] P. Karki and K. G. Birukov, "Lipid mediators in the regulation of endothelial barriers," Tissue Barriers, vol. 6, no. 1, Article ID e1385573, 2018.

[5] Y. Zhu, X. Xian, Z. Wang et al., "Research progress on the relationship between atherosclerosis and inflammation," Biomolecules, vol. 8, no. 3, 2018.

[6] Y. Takafuji, M. Hori, T. Mizuno, and M. Harada-Shiba, "Humoral factors secreted from adipose tissue-derived mesenchymal stem cells ameliorate atherosclerosis in Ldlr-/mice," Cardiovascular Research, vol. 115, no. 6, pp. 1041-1051, 2019.

[7] F. Lin, L. Pei, Q. Zhang et al., "Ox-LDL induces endothelial cell apoptosis and macrophage migration by regulating caveolin-1 phosphorylation," Journal of Cellular Physiology, vol. 233, no. 10, pp. 6683-6692, 2018.

[8] M. Zhang and L. Jiang, "Oxidized low-density lipoprotein decreases VEGFR2 expression in HUVECs and impairs angiogenesis," Experimental and Therapeutic Medicine, vol. 12, no. 6, pp. 3742-3748, 2016.

[9] C. Zeng, R. Wang, and H. Tan, "Role of pyroptosis in cardiovascular diseases and its therapeutic implications," International Journal of Biological Sciences, vol. 15, no. 7, pp. 1345-1357, 2019.

[10] L. Galluzzi, I. Vitale, J. M. Abrams et al., "Molecular definitions of cell death subroutines: recommendations of the nomenclature committee on cell death 2012," Cell Death \& Differentiation, vol. 19, no. 1, pp. 107-120, 2012.

[11] S. Toldo and A. Abbate, "The NLRP3 inflammasome in acute myocardial infarction," Nature Reviews Cardiology, vol. 15, no. 4, pp. 203-214, 2017.

[12] P. Duewell, H. Kono, K. J. Rayner et al., "NLRP3 inflammasomes are required for atherogenesis and activated by cholesterol crystals," Nature, vol. 464, no. 7293, pp. 1357-1361, 2010.

[13] V. Ambros, "The functions of animal microRNAs," Nature, vol. 431, no. 7006, pp. 350-355, 2004.

[14] D. Foteini, T. Margaritis, A. Panagiotis, K. Levis, and T. Tokas, "MiR-221/222 cluster expression improves clinical stratification of non-muscle invasive bladder cancer (TaT1) patients' risk for short-term relapse and progression," Genes Chromosomes \& Cancer, vol. 57, no. 3, pp. 150-161, 2018.

[15] F. Q. Long, C. X. Kou, K. Li, J. Wu, and Q. Q. Wang, "MiR223-3p inhibits rTp17-induced inflammasome activation and pyroptosis by targeting NLRP3," Journal of Cellular and Molecular Medicine, vol. 24, no. 24, pp. 14405-14414, 2020.

[16] F. Bauernfeind, A. Rieger, F. A. Schildberg, P. A. Knolle, J. L. Schmid-Burgk, and V. Hornung, "NLRP3 inflammasome activity is negatively controlled by miR-223," The Journal of Immunology, vol. 189, no. 8, pp. 4175-4181, 2012.

[17] X. Bai, Y. Zuo, G. Luo, and S. J. Yang, "Effects of Zhilonghuoxuetongyu Capsules on inflammatory cytokines in rats with cerebral ischemia and reperfusion," Western Medicine, vol. 21, no. 011, pp. 1847-1849, 2009, [In Chinese].

[18] Y. F. Yang, J. Huang, S. J. Yang et al., "Effects of Zhilonghuoxuetongyu Capsules combined with enalapril maleate folic acid tablets on risk factors of stroke in patients with type $\mathrm{H}$ hypertension," Journal of Southwest Medical University, vol. 42, no. 4, pp. 345-347, 2019, [In Chinese].

[19] L. Dong, B. Li, X. Bai, D. C. Zhang, and S. J. Yang, "Effect of Zhilonghuoxuetongyu Capsules on acutecerebral edema and hs-CRP in patients with ischemic stroke," Journal of Luzhou Medical College, vol. 38, no. 2, p. 180, 2015 [In Chinese].

[20] M. N. Liu, M. Han, S. J. Yang, W. Ren, and P. Liu, "Effects of Zhilonghuoxuetongyu Capsules on NLRP3 and TGF- $\beta$ in rabbits with hyperlipidemia and carotid atherosclerosis," Journal of Southwest Medical University, vol. 43, no. 4, pp. 329-334, 2020, [In Chinese].

[21] L. Yang, L. Tian, Z. Zhang et al., "Cannabinoid receptor 1/ miR-30b-5p Axis governs macrophage NLRP3 expression and inflammasome activation in liver inflammatory disease," Molecular Therapy-Nucleic Acids, vol. 20, pp. 725-738, 2020.

[22] X. Zhong, L. Zhang, Y. Li, P. Li, J. Li, and G. Cheng, "Kaempferol alleviates ox-LDL-induced apoptosis by upregulation of miR-26a-5p via inhibiting TLR4/NF- $\kappa$ B pathway in human endothelial cells," Biomedicine \& Pharmacotherapy, vol. 108, pp. 1783-1789, 2018.

[23] O. J. Lara-Guzmán, Á. Gil-Izquierdo, S. Medina et al., "Oxidized LDL triggers changes in oxidative stress and inflammatory biomarkers in human macrophages," Redox Biol, vol. 15, pp. 1-11, 2018.

[24] Z. Fu, E. Zhou, X. Wang et al., "Oxidized low-density lipoprotein-induced microparticles promote endothelial monocyte adhesion via intercellular adhesion molecule 1," American Journal of Physiology-Cell Physiology, vol. 313, no. 5, pp. C567-c574, 2017.

[25] Z. Wang, X. Guo, Q. Zhang et al., "Elimination of Ox-LDL through the liver inhibits advanced atherosclerotic plaque progression," International Journal of Medical Sciences, vol. 18, no. 16, pp. 3652-3664, 2021.

[26] P. Liang, L. Mao, Y. Ma, W. Ren, and S. Yang, "A systematic review on Zhilong Huoxue Tongyu capsule in treating cardiovascular and cerebrovascular diseases: pharmacological actions, molecular mechanisms and clinical outcomes," Journal of Ethnopharmacology, vol. 277, p. 114234, 2021.

[27] M. Liu, Y. Pu, J. Gu et al., "Evaluation of Zhilong Huoxue Tongyu capsule in the treatment of acute cerebral infarction: a systematic review and meta-analysis of randomized controlled trials," Phytomedicine, vol. 86, Article ID 153566, 2021.

[28] J. W. V. G. B. Fenton, F. A. Ofosu, and J. M. Maraganore, "Thrombin inhibition by hirudin: how hirudin inhibits thrombin," Haemostasis, vol. 21, no. Suppl 1, pp. 27-31, 1991.

[29] J. D. Welsh, I. Poventud-Fuentes, S. Sampietro, S. L. Diamond, T. J. Stalker, and L. F. Brass, "Hierarchical 
organization of the hemostatic response to penetrating injuries in the mouse macrovasculature," Journal of Thrombosis and Haemostasis, vol. 15, no. 3, pp. 526-537, 2017.

[30] N. Reglero-Real, B. Colom, J. V. Bodkin, and S. Nourshargh, "Endothelial cell junctional adhesion molecules," Arteriosclerosis, Thrombosis, and Vascular Biology, vol. 36, no. 10, pp. 2048-2057, 2016.

[31] M. W. Majesky, "Vascular smooth muscle cells," Arteriosclerosis, Thrombosis, and Vascular Biology, vol. 36, no. 10, pp. e82-6, 2016.

[32] T. D. Manes and J. S. Pober, "Significant differences in antigen-induced transendothelial migration of human CD8 and CD4 T effector memory cells," Arteriosclerosis, Thrombosis, and Vascular Biology, vol. 36, no. 9, pp. 1910-1918, 2016.

[33] P. F. Davies, "Hemodynamic shear stress and the endothelium in cardiovascular pathophysiology," Nature Clinical Practice Cardiovascular Medicine, vol. 6, no. 1, pp. 16-26, 2009.

[34] X. Li, N. Du, Q. Zhang et al., "MicroRNA-30d regulates cardiomyocyte pyroptosis by directly targeting foxo3a in diabetic cardiomyopathy," Cell Death \& Disease, vol. 5, no. 10, Article ID e1479, 2014.

[35] W. Chang, J. Lin, J. Dong, and D. Li, "Pyroptosis: an inflammatory cell death implicates in atherosclerosis," Medical Hypotheses, vol. 81, no. 3, pp. 484-486, 2013.

[36] G. K. Gifford, A. J. Gifford, Q. Chen et al., "Fatty acid synthase and adenosine monophosphate-activated protein kinase regulate cell survival and drug sensitivity in diffuse large B-cell lymphoma," Leukemia \& Lymphoma, vol. 61, no. 8, pp. 1810-1822, 2020.

[37] X. Peng, H. Chen, Y. Li, D. Huang, B. Huang, and D. Sun, "Effects of NIX-mediated mitophagy on ox-LDL-induced macrophage pyroptosis in atherosclerosis," Cell Biology International, vol. 44, no. 7, pp. 1481-1490, 2020.

[38] Y. Li, X. Niu, H. Xu et al., "VX-765 attenuates atherosclerosis in ApoE deficient mice by modulating VSMCs pyroptosis," Experimental Cell Research, vol. 389, no. 1, Article ID 111847, 2020.

[39] E. A. Miao, J. V. Rajan, and A. Aderem, "Caspase-1-induced pyroptotic cell death," Immunological Reviews, vol. 243, no. 1, pp. 206-214, 2011.

[40] V. Sagulenko, S. J. Thygesen, D. P. Sester et al., "AIM2 and NLRP3 inflammasomes activate both apoptotic and pyroptotic death pathways via ASC," Cell Death \& Differentiation, vol. 20, no. 9, pp. 1149-1160, 2013.

[41] E. A. Miao, I. A. Leaf, P. M. Treuting et al., "Caspase-1-induced pyroptosis is an innate immune effector mechanism against intracellular bacteria," Nature Immunology, vol. 11, no. 12, pp. 1136-1142, 2010.

[42] X. Qin and J. Guo, "MicroRNA-328-3p protects vascular endothelial cells against oxidized low-density lipoprotein induced injury via targeting forkhead box protein O4 (FOXO4) in atherosclerosis," Medical Science Monitor, vol. 26, Article ID e921877, 2020.

[43] M.-N. Liu, G. Luo, W.-J. Gao, S.-J. Yang, and H. Zhou, “miR29 family: a potential therapeutic target for cardiovascular disease," Pharmacological Research, vol. 166, Article ID 105510, 2021.

[44] C. C. Woo, W. Liu, X. Y. Lin et al., "The interaction between 30b-5p miRNA and MBNL1 mRNA is involved in vascular smooth muscle cell differentiation in patients with coronary atherosclerosis," International Journal of Molecular Sciences, vol. 21, no. 1, 2019.

[45] X. Qi, H. Wang, L. Xia et al., "miR-30b-5p releases HMGB1 via UBE2D2/KAT2B/HMGB1 pathway to promote pro- inflammatory polarization and recruitment of macrophages," Atherosclerosis, vol. 324, pp. 38-45, 2021.

[46] E. Kaniewska-Bednarczuk, M. Mielcarek, A. H. Chester, E. M. Slominska, M. H. Yacoub, and R. T. Smolenski, "Oxidized low-density lipoproteins enhance expression and activity of CD39 and CD73 in the human aortic valve endothelium," Nucleosides, Nucleotides \& Nucleic Acids, vol. 35, no. 10-12, pp. 713-719, 2016.

[47] H. Xu, J. Zhang, X. Shi, X. Li, and C. Zheng, "NF- $\kappa$ B inducible miR-30b-5p aggravates joint pain and loss of articular cartilage via targeting SIRT1-FoxO3a-mediated NLRP3 inflammasome," Aging, vol. 13, no. 16, pp. 20774-20792, 2021.

[48] W. Zeng, D. Wu, Y. Sun et al., "The selective NLRP3 inhibitor MCC950 hinders atherosclerosis development by attenuating inflammation and pyroptosis in macrophages," Scientific Reports, vol. 11, no. 1, p. 19305, 2021.

[49] R. Yin, X. Zhu, J. Wang et al., "MicroRNA-155 promotes the ox-LDL-induced activation of NLRP3 inflammasomes via the ERK1/2 pathway in THP-1 macrophages and aggravates atherosclerosis in ApoE-/- mice," Annals of Palliative Medicine, vol. 8, no. 5, pp. 676-689, 2019. 